\title{
Action matters: The role of action plans and object affordances in selection for action
}

\author{
Antonella Pavese \\ Moss Rehabilitation Research Institute, Philadelphia, PA, USA
}

Laurel J. Buxbaum

Moss Rehabilitation Research Institute and Thomas Jefferson University, Philadelphia, PA, USA

In a series of three experiments requiring selection of real objects for action, we investigated whether characteristics of the planned action and/or the "affordances" of target and distractor objects affected interference caused by distractors. In all of the experiments, the target object was selected on the basis of colour and was presented alone or with a distractor object. We examined the effect of type of response (button press, grasping, or pointing), object affordances (compatibility with the acting hand, affordances for grasping or pointing), and target/distractor positions (left or right) on distractor interference (reaction time differences between trials with and without distractors). Different patterns of distractor interference were associated with different motor responses. In the button-press conditions of each experiment, distractor interference was largely determined by perceptual salience (e.g., proximity to initial visual fixation). In contrast, in tasks requiring action upon the objects in the array, distractors with handles caused greater interference than those without handles, irrespective of whether the intended action was pointing or grasping. Additionally, handled distractors were relatively more salient when their affordances for grasping were strong (handle direction compatible with the acting hand) than when affordances were weak. These data suggest that attentional highlighting of specific target and distractor features is a function of intended actions.

Please address all correspondenc e to L. Buxbaum, Moss Rehabilitation Research Institute, 1200 W. Tabor Rd., Philadelphia, PA 19141, USA. Email: Lbuxbaum@aehn2.einstein.ed u

We are grateful to Myrna Schwartz and John Duncan for their contribution s to experimental design and data interpretation. Perdita Permaul, Ivan Kautter, and Adrian Wilson are to be thanked for running subjects and assisting with data analysis. Supported by NIH grants R29 NS36387 and RO1 NS31824. 
Cognitive processes involved in selection of objects for action have been widely and successfully investigated in experimental psychology (Allport, 1987). For the most part, the strategy has been to limit the range of object features and action features that are experimentally manipulated and to use artificial (usually two-dimensional) environments that permit control over experimental variables and precise measurement of dependent variables (usually reaction times or errors). In many instances, as in studies of visual search processes, for example, this strategy is effective and appropriate in reducing computational complexity of the problem and permitting isolation of the variables that influence visual selection. In studies involving attentional selection for action, however, such experimental simplification necessarily depends upon specific assumptions about which factors are relevant for object and action selection and which are not. In turn, the choice of variables that are investigated influences experimental results and the theories based on these results.

An instructive example comes from the debate on stimulus-response (S-R) compatibility effects. The influential coding theory of S-R compatibility (Kornblum, Hasbroucq, \& Osman, 1990; Proctor \& Reeve, 1990) states that S-R compatibility effects arise from the consistency of stimulus and response codes. This approach proposes that both the stimulus and the response are represented as abstract mental codes and that the appropriate response is selected via a process of S-R translation of these codes (Proctor, Van Zandt, Lu, \& Weeks, 1993). Therefore, specific and concrete parameters of action (such as the type and extent of movement required to access the target) are unimportant in the coding theory. In keeping with this view (and reinforcing it), S-R compatibility has traditionally been studied by using button-press responses to demonstrate compatibility between location of stimulus and location of response (Proctor \& Reeve, 1990).

Ecological approaches, in contrast, hold that S-R compatibility phenomena reflect the extent to which available information can guide action. On this approach, a situation is "compatible" if the information inherent in an object or array is appropriate to the needs of the physical programming of a co-ordinated action (Michaels \& Stins, 1997). Objects directly afford a number of possible actions as a joint function of object characteristics (e.g., shape, size, weight, texture, and location) and organism attributes (e.g., size and type of effectors, type of movements, motivation; Gibson, 1979). Because the influence of affordances emerges in tasks that require direct action upon objects in the array, the ecological perspective holds that such tasks are necessary if the relationships between objects, goals, and actions are to be well understood.

In the next section, we will discuss several recent results that suggest that the relationship between action parameters, affordances, and object features influences selection for action. 


\section{EFFECT OF AFFORDANCES AND ACTION PARAMETERS ON SELECTION}

Three characteristics of traditional selective attention paradigms limit their relevance for the study of attention and action. First, the stimuli to be selected are frequently symbolic or simple visual elements, which do not have affordances for action. Second, participants locate or identify target objects presented in a visual display, but respond by performing an unrelated action directed to locations other than the targets. For example, they may select a red shape on the computer monitor, and respond by pressing a key on the computer keyboard. Third, the mapping of this action is arbitrary and does not correspond in any natural way to features present in the object. All three factors minimize the possibility of observing relationships between affordances and action parameters in selective attention tasks.

Recently, there has been increased interest in studying selection for action in more naturalistic contexts (e.g., Castiello, 1996). Under such circumstances, the influence of affordances may begin to emerge. For example, Tucker and Ellis (1998) showed that subjects' responses to the orientation of an object were influenced by task-irrelevant affordances of the object. Subjects viewed photographs of upright and inverted objects which had handles on the left or right. On one experimental trial, for example, subjects viewed a frying pan, oriented upside-down, whose handle was on the right of the photograph. Subjects responded by a button press with one hand if the photographed object was upright, and with the other hand if it was inverted. The task-irrelevant feature (handle direction) resulted in significant compatibility effects: Left-hand responses were faster when handles were on the left, and right-hand responses were faster when handles were on the right. In contrast, this effect was not observed when participants used left and right fingers of a single hand. This suggests that the photograph of the object activated the hand appropriate to object grasping. In this experiment, the response did not require reaching for the target object, and the mapping between target feature (object orientation) and response (left or right button press) was arbitrary. However, the use of photographs of complex, real objects (rather than, say, abstract shapes) revealed the presence of automatic activation of responses based on object affordances.

Similar conclusions emerged from an interesting study by Riddoch, Edwards, Humphreys, West, and Heafield (1998) that examined action on objects in a patient with impairment of hand control associated with corticobasal degeneration. In one experiment, the task requirement was to grasp cups presented on the left of the array with the left hand and cups presented on the right with the right hand. The position of the cup handle was the crucial variable. In half of the trials the position of the cup and the position of the handle were compatible (e.g., the cup was on the right, requiring a right- 
hand response, and the handle faced right), and in half of the trials they were incompatible (e.g., the cup was on the right and the handle faced left). The patient's performance was strongly influenced by handle compatibility. She responded with the correct hand on $98 \%$ of the trials in the compatible condition, but only $10 \%$ of the trials in the incompatible condition. The studies of Riddoch et al. (1998) and Tucker and Ellis (1998) provide converging evidence that the presence of a handle in the target object activates responses with the ipsilateral hand.

Compelling evidence for the importance of action parameters (e.g., type of manual response) in selection for action comes from experiments that require reaching and pointing to a target stimulus rather than pressing an unrelated key. A seminal series of studies by Tipper and colleagues (Meegan \& Tipper, 1998, 1999; Tipper, Howard, \& Jackson, 1997; Tipper, Lortie, \& Baylis, 1992) revealed that distractor interference is influenced by the position of the distractor relative to the effector. Stimuli were pairs of LEDs of different colours, and participants were asked to reach to and touch the target stimulus (red LED), ignoring the distractor stimulus (yellow LED). They demonstrated four important effects. First, distractors close to the acting hand yielded more interference than distractors further from the acting hand, even when they were not on the reaching path (Meegan \& Tipper, 1998; Tipper et al., 1992). Second, when distance was controlled, distractors on the same side of the array as the acting hand yielded more interference than distractors on the opposite side. Third, the trajectory of the reaching movement was influenced by the presence of distractors even when they did not represent a physical obstacle to the movement. Fourth, the interfering potential of a distractor depended on the relative ease of programming a movement toward that distractor as compared to the target. Interference from distractors that were covered by a transparent obstacle was reduced, whereas when the target was covered by a transparent obstacle, interference from distractors was increased (Meegan \& Tipper, 1999). These effects were only observed when a reaching response was required, and not when the response was verbal (Meegan \& Tipper, 1999).

Tipper et al. (1992) proposed that attention to the array (and hence, object selection) is action-centred (rather than retinotopic or body-centred) when responses require action upon objects in the array. This proposal assumes that perception and action are inter-dependent (Gibson, 1979; Shaw \& Turvey, 1981), and that what is attended and perceived depends on the action to be performed.

Two important conclusions emerge from the literature cited. First, the effect of affordances can be observed when familiar objects, rather than symbols or simplified shapes, are used as stimuli. Second, the effects of action parameters (e.g., effector side, distance between effector and stimuli, type of response) can be observed when the task requires reaching for the target stimulus, rather than pressing a button associated with the target feature by an arbitrary rule. 


\section{OVERVIEW}

In this study, our goal was to examine the influence of affordances and action parameters on selection for action. To maximize the possibility that we would observe affordance effects, we used familiar objects (cups with and without handles in Experiments 1 and 2; drawer handles, doorbells, and light-switches in Experiment 3) associated naturalistically with grasping and pointing responses. To examine the effect of action parameters, we varied the type of motor response required. Each experiment had a button-press version and a reaching version; in Experiments 2 and 3, the reaching version called for both pointing and grasping responses. In all three experiments, the dependent variable was interference (reaction time differences between a distractor present condition and a distractor absent condition).

In Experiment 1, we tested a naturalistic extension of the proposal by Meegan and Tipper (1999) that the interference caused by distractors is a function of the relative ease of programming an action to the distractors versus the targets. We assessed the hypothesis that in a task requiring reaching to and grasping a target cup, the interference caused by distractors with handles would be modulated by the position of the handle relative to the responding hand. In Experiment 2, we tested the hypothesis that the type of reaching response to target cups (pointing vs grasping) may influence the interference caused by distractor cups with and without handles. In Experiment 3, we assessed whether affordance-related interference effects are enhanced when objects (handles and buttons) strongly afford their associated actions (grasping and pointing, respectively) and when these actions can be pre-programmed.

\section{GENERAL METHODS}

\section{Apparatus}

The display apparatus was a wooden structure (dimensions: 21 " height $\times 23$ " width $\times 8$ " depth) with four platforms (Figure 1). The display platforms were $4 "$ wide $\times 4$ " deep and were fitted with touch-sensitive microswitches. Only the two lower platforms, which were mounted 2 inches from the base of the apparatus and 6 inches apart from each other, were used in the present study. Portable visual occlusion spectacles (PLATO, Translucent Technologies, Inc.) were used to control stimulus presentation time. The lenses of these goggles can rapidly (about $5 \mathrm{~ms}$ ) switch from their light scattering, occluding state to their transparent state, during which $90 \%$ of incident light is transmitted. The display platform, goggles, and a start button were all connected to a PC. The computer was controlled by a custom program that timed the trials, played auditory stimuli, and recorded responses. 


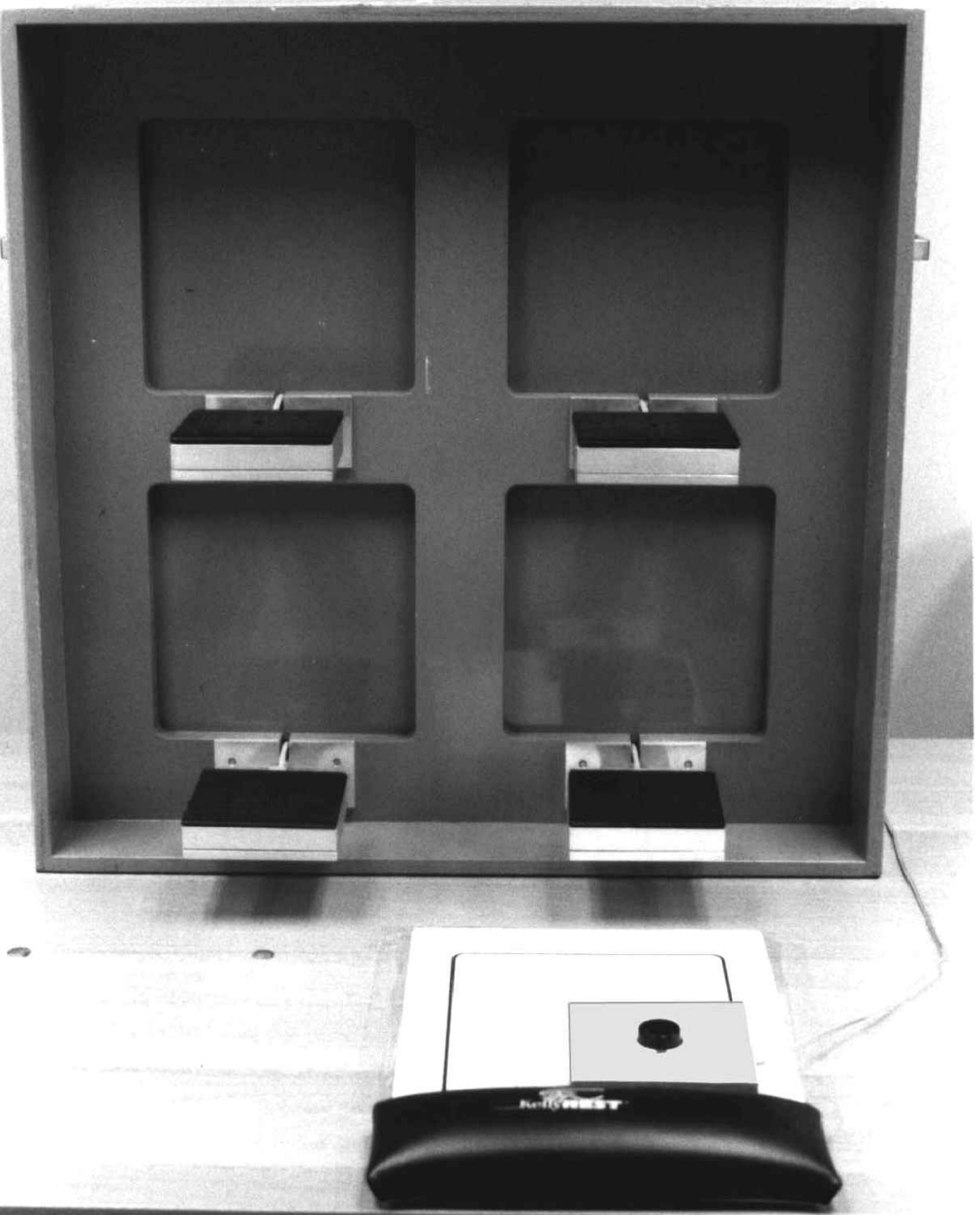

Figure 1. The apparatu s used for stimulus presentation. The photograph also shows the start key used to control for the starting position of the hand and to record initiation times. The start key is positioned in front of the right platform.

To control the start position in each trial and to obtain a measure of movement initiation time, participants started each trial by pressing a button on a keypad. In the reaching conditions, the start button was a single round button in the centre of a square keypad. In the button-press conditions, the keypad had two response keys. The start button was a square button in the centre of the pad and the two response buttons were placed to the right of the start button, $2 \mathrm{~cm}$ above and $2 \mathrm{~cm}$ below the start button position. 
A description of the objects used as stimuli is provided in the Methods sections of the individual experiments.

\section{Participants}

Participants in these experiments were right-handed older adults. Some individuals participated in more than one experiment. All had normal or corrected to normal vision and normal colour vision. Fourteen individuals (ten women) participated in Experiment 1. Their average age was 59.2 years (range 45-76) and the average education was 13.4 years. Fourteen individuals (seven women) participated in Experiment 2. Their average age was 56.5 (range 39-81) and the average education was 13.2 years. Finally, 18 individuals participated in Experiment 3 (11 women). Their average age was 64.1 (range 29-83) and the average education was 13.7 years. ${ }^{1}$

\section{Procedure}

Participants were seated directly in front of the display platform with the centre of the platform at midline. This placed the two lower platforms approximately 11 inches away from the body wall, approximately 10 inches below eye level (when the subjects looked straight ahead), and at $20^{\circ}$ and $70^{\circ}$ from the body midline. Before each trial, with the goggles in the occluded state, the experimenter placed the stimulus objects on the platforms. To begin each trial, the participants pushed the start button, which was placed to the right of midline, directly in front of the right platform. ${ }^{2}$ The experimenter started the trial after determining that the participant was ready, was seated with head and body midline, and was pushing the start button. A tone was played and, after $500 \mathrm{~ms}$, the goggles cleared. Participants were asked to respond to the target stimulus, selected by colour (blue). In the button-press task, participants were asked to press one of two buttons corresponding to a target feature (e.g., press the top button if the handle of the blue cup faces right and press the bottom button if the handle faces left). To avoid stimulus-response compatibility effects, the two response keys were organized vertically and the key assignment was

\footnotetext{
${ }^{1}$ The subjects run in these experiment s are, on average, older than those commonly used in the experimental literature, and their ages span a greater range. Investigations in our laboratory frequently centre on patients aged 30-80 who have suffered strokes. To leave open the option of studies comparing healthy and brain-damage d subjects, we often recruit healthy, active subjects from a population that is age-matched to our stroke population.

${ }^{2} \mathrm{We}$ chose this right-of-midline start position to maximize the possibility of observing proximity-to-hand distractor interference effects.
} 
switched in the middle of each experiment. In grasping tasks, participants were asked to reach to and grasp the target object. In pointing tasks, participants were asked to reach and point to the target objects, perturbing them slightly with a light touch. The instructions specified that responses were to be executed as quickly as possible. Initiation time was recorded as the latency between goggles clearing and the point at which the hand was lifted from the keypad. Movement time was recorded as the latency between the hand lifting from the keypad and the point at which an object on one of the platforms was perturbed. When the subject made the correct response, a short (100 ms) beeptone was played. If the subject responded to the distractor object, an external buzzer was played for $500 \mathrm{~ms}$. If the subject failed to respond within $10 \mathrm{~s}$, initiation and movement times were not recorded, and a different long tone was played.

\section{Dependent variable and experimental design}

Interference scores served as dependent variable in the three experiments. After discarding trials with RTs longer than $2000 \mathrm{~ms}$, mean RTs were computed for the distractor present and distractor absent conditions for each subject and each condition. The $2000 \mathrm{~ms}$ cut-off led to the elimination of 115 trials out of 21,120 total trials $(0.5 \%)$. Interference scores were computed by subtracting the no-distractor condition from the corresponding distractor condition. This procedure allowed us to subtract out variability in RTs due to differences in reaching to the two target positions (left or right) or differences in motor response (e.g., grasping vs pointing). For example, we would expect faster responses to targets on the right, because the starting position was closer to the right than left stimulus pad. However, any observed differences in interference with right versus left distractors would not be attributable to the different trajectories required to reach the target.

We chose to use interference scores computed from total RT (from clearing of the goggles to contact with stimuli) rather than from initiation RT because the moment at which participants released the start button could be influenced by strategy. Because there were no catch trials in which participants were asked to withhold responses, there was no motivation for participants to delay release of the start button until object selection was complete. Indeed, it has been reported that in such conditions, participants often release the start button before the selection process is completed (see Meegan \& Tipper, 1998, for a detailed discussion of this issue).

The experimental design was similar for the three experiments. Each study had four within-subjects factors: response type, target compatibility, distractor compatibility, and distractor position. The levels of each variable were different in the three experiments, and they are specified in the Methods sections of each experiment. 


\section{EXPERIMENT 1 \\ Effects of handle-hand compatibility}

The first experiment examined whether the affordances of distractor objects affect responses to target objects. The findings by Riddoch et al. (1998) and Tucker and Ellis (1998), discussed earlier, demonstrated that affordances of the target stimulus influence responses even when irrelevant. In particular, these studies have shown that (1) the presence of a handle in a target object automatically activates responses with the hand, and (2) responses with the hand are particularly facilitated when the handle is oriented toward the hand as compared to when the handle is oriented in the opposite direction.

These results suggest the possibility that the interference caused by distractor objects may be modulated by the degree to which the distractors afford actions by the responding hand. In particular, interference caused by distractors with handles may be a function of the position of the handles relative to the acting hand. We expect distractor cups to be more interfering when their handles are oriented toward the responding hand as compared to when their handles are oriented away from the responding hand. Furthermore, this effect should be found when the task requires participants to grasp the cup handle, but not when participants are asked to press a button arbitrarily associated with the handle attribute of the target cup. We also expect to observe the proximity-to-hand effect found by Tipper et al. (1992); that is, to find greater interference from distractors close to the acting hand as compared to distractors farther from the acting hand. In the button-press task, we do not expect affordance-related or action-centred interference effects. Instead, we expect that other variables (for example, perceptual salience) should influence interference.

To test these hypotheses, we asked participants to respond with their right hands to target cups selected on the basis of colour. In most trials, a distractor cup of a different colour was also presented. Both target and distractor cups could have their handles facing right (good affordance for grasping) or left (bad affordance for grasping). In both the grasp and button-press tasks, the details of the response were a function of stimulus attributes. In the grasping task, participants had to grasp the handle of the target cup, and parameters of the grasp (e.g., degree of wrist flexion) were influenced by whether the handle faced right or left. In the button-press task, participants had to press one button if the target handle faced right and another button if it faced left.

\section{Methods}

Stimuli. The stimuli were ceramic cups 5 inches high and with a diameter of 4 inches (Figure 2). Cup handles protruded 2 inches horizontally and had a vertical opening of about 2 inches. The cups were painted dark blue and dark purple. Colours were piloted to insure that selection on the basis of colour was not unduly easy. 


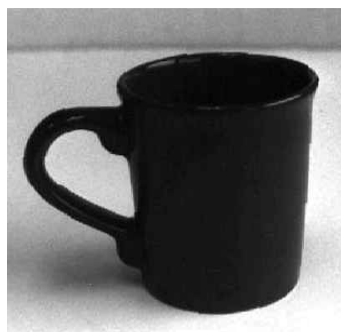

A

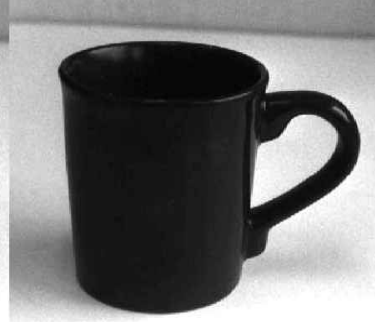

B

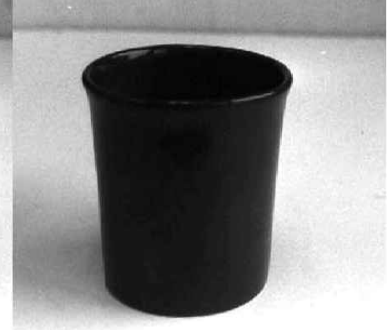

C

Figure 2. Cups used as stimuli in Experiments 1 and 2. A (cups with handle facing left) and B (cups with handle facing right), were the two stimulus configurations used in Experiment 1. B (cups with handle facing right) and $\mathrm{C}$ (cups without handle) were the two stimulus configurations used in Experiment 2.

Procedure. The target cup (blue) was presented either on the left or on the right lower pad of the display apparatus, with the handle facing right (compatible condition) or left (incompatible condition). Participants always responded with the right hand. There were three distractor compatibility conditions: Nodistractor, distractor with handle facing right (compatible distractor), and distractor with handle facing left (incompatible distractor). The no-distractor condition was used as a baseline to compute interference. Therefore, there were 12 cells for each response type (grasp and button press): 2 Positions $\times 2$ Target compatibilities $\times 3$ Distractor compatibilities.

The experimental session was divided in four 96-trial blocks, two grasping blocks and two button-press blocks. The two blocks for each task were run consecutively. The order of the two tasks (grasp first vs button press first) was counterbalanced across subjects. A short block of practice trials was presented before each task. During the entire session, participants completed 384 experimental trials, 16 for each of the 24 cells in the design.

\section{Results}

Table 1 shows the mean response latencies in distractor and no-distractor conditions in this experiment. The results of Experiment 1 were analysed in a four-way repeated measures ANOVA that included the following factors: (1) Response type (button press vs grasping), (2) target compatibility (compatible and incompatible), (3) distractor compatibility (compatible and incompatible), and (4) distractor position (left and right). The dependent variable was interference (total RT in each of the distractor-present conditions minus total RT in the equivalent distractor-absent condition). Significance values of post hoc comparisons were calculated using two-tailed paired $t$-tests. 
TABLE 1a

Mean response latencies in distractor and no-distractor conditions of Experiment 1

Button press

Right Left

No

Target type Compatible Incompatible distractor

No

Compatible Incompatible distractor

Target

compatible

995

1073

928

970

976

878

Target

incompatible

1012

1044

941

1041

995

911

TABLE 1b

\begin{tabular}{|c|c|c|c|c|c|c|}
\hline \multirow[b]{3}{*}{ Target type } & \multicolumn{6}{|c|}{ Grasp } \\
\hline & \multicolumn{3}{|c|}{ Right } & \multicolumn{3}{|c|}{ Left } \\
\hline & Compatible & Incompatible & $\begin{array}{c}\text { No } \\
\text { distractor }\end{array}$ & Compatible & Incompatible & $\begin{array}{c}\text { No } \\
\text { distractor }\end{array}$ \\
\hline $\begin{array}{l}\text { Target } \\
\text { compatible }\end{array}$ & 1003 & 989 & 952 & 954 & 925 & 908 \\
\hline $\begin{array}{l}\text { Target } \\
\text { incompatible }\end{array}$ & 1093 & 1079 & 1050 & 958 & 933 & 894 \\
\hline
\end{tabular}

Compatible $=$ handle on the right; incompatible $=$ handle on the left.

The analysis showed a significant effect of response type, $F(1,13)=18.72$, $M S e=10024.33, p<.001$. Interference was greater in the button-press task than in the grasping task (99 and $41 \mathrm{~ms}$, respectively).

Response type interacted with distractor compatibility, $F(1,13)=16.24$, $M S e=11230.77, p<.002$. In the grasping task, interference was greater in the compatible distractor condition than in the incompatible distractor condition (51 and $31 \mathrm{~ms}$, respectively, $p<.005$ ). In the button-press task, the opposite pattern was observed: Interference was smaller in the compatible distractor condition than in the incompatible distractor condition (90 and $108 \mathrm{~ms}$, respectively, $p<.05$ ).

Two three-way interactions involving response type were also significant: Response type $\times$ Distractor compatibility $\times$ Target compatibility, $F(1,13)=$ $6.59, M S e=1342.99, p<.025$, and Response type $\times$ Distractor compatibility $\times$ Distractor position, $F(1,13)=5.12, M S e=2751.65, p<.05$. To explore the Response type $\times$ Distractor compatibility $\times$ Distractor position interaction, two separate ANOVAs were carried out on the button-press and grasping data, 
revealing that the Target compatibility $\times$ Distractor compatibility interaction was significant in the button-press task, $F(1,13)=5.72, p<.03$, but not in the grasping task $(F<1, p>.8)$. In the grasping task, compatible distractors yielded more interference than incompatible distractors, regardless of the type of target: For compatible targets: $49 \mathrm{vs} 27 \mathrm{~ms}, t(13)=2.1, p=.05$; for incompatible targets: 54 vs $34 \mathrm{~ms}, t(13)=2.8, p=.01$. In contrast, in the button-press task, incompatible distractors were relatively more interfering than compatible distractors (122 vs $80 \mathrm{~ms}$ ) when the target was compatible, $t(13)=2.8 p=.01$; and compatible distractors were slightly, though not significantly, more interfering when the target was incompatible (101 vs $94 \mathrm{~ms}, t(13)=0.7, p=.4$ ) (Figures 3 and 4).

To further explore the Response type $\times$ Distractor compatibility $\times$ Distractor position interaction, we performed two separate ANOVAs on the button-press and grasping data. These revealed that the target compatibility $\times$ distractor position interaction was significant in the button press task, $F(1,13)=12.40$, $p<.005$, but not in the grasping task $(F<1, p>.35)$. In the button-press task, compatible distractors were more interfering when presented on the left, $t(13)=$ $-4.67, p<.0005$, and incompatible distractors tended to be more interfering when presented on the right, although not significantly so, $t(13)=1.53, p=.1$. In the grasping task this trend was arguably still present but dramatically reduced (Figures 5 and 6).

The Target compatibility $\times$ Distractor position interaction approached significance, $F(1,13)=4.26, M S e=2861.05, p<.06$. This interaction

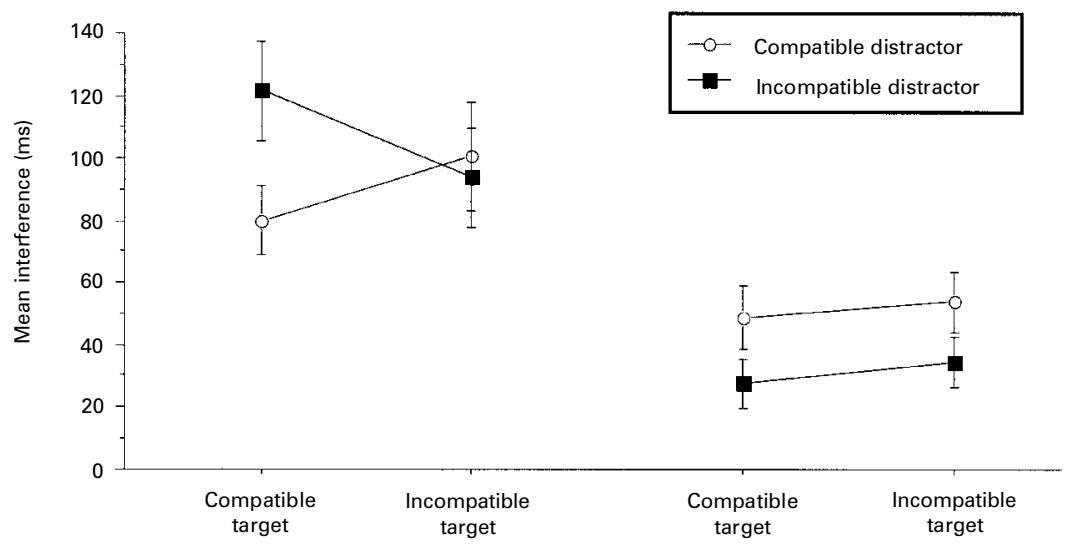

Button press response

Grasping response

Figure 3. Interference in ms (and standard error) as a function of response type (button press and grasping), distractor compatibility (compatible and incompatible), and target compatibility (compatible and incompatible) in Experiment 1. 

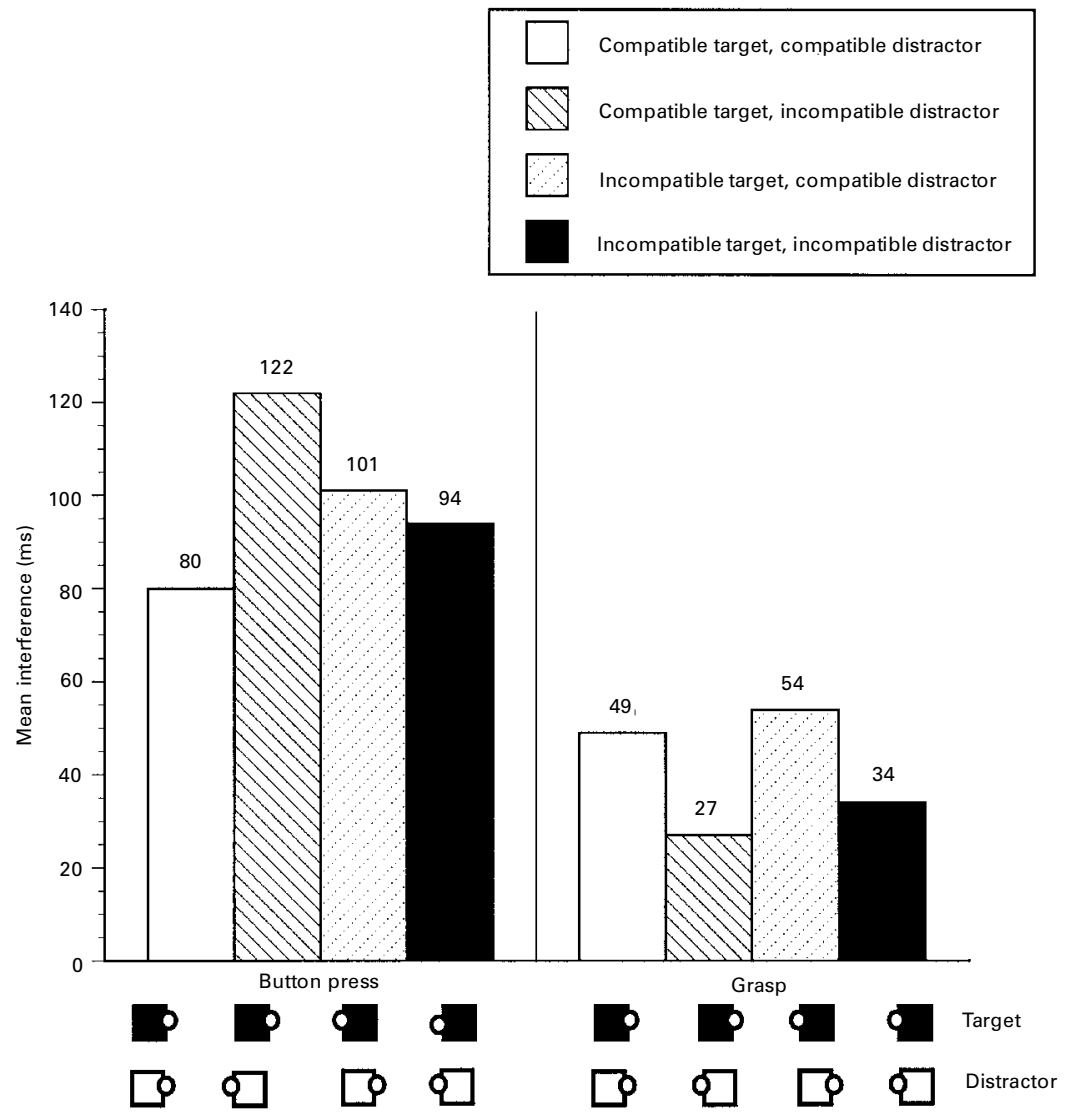

Figure 4. Interference in Experiment 1 as a function of response type, target compatibility, and distractor compatibility, shown with graphic representations of stimulus configurations in each condition.

indicated that compatible targets tended to receive more interference from right distractors than left distractors (75 vs $64 \mathrm{~ms}$ ), whereas the opposite was true for incompatible targets (62 vs $79 \mathrm{~ms}$ ).

\section{Discussion}

Experiment 1 showed that button-press and grasping tasks differ in the pattern of interference caused by distractors. First, interference effects were generally greater in the button-press task than in the grasping task. More importantly, in the grasping task compatible distractors yielded more interference than incompatible distractors, whereas in the button-press task, incompatible 


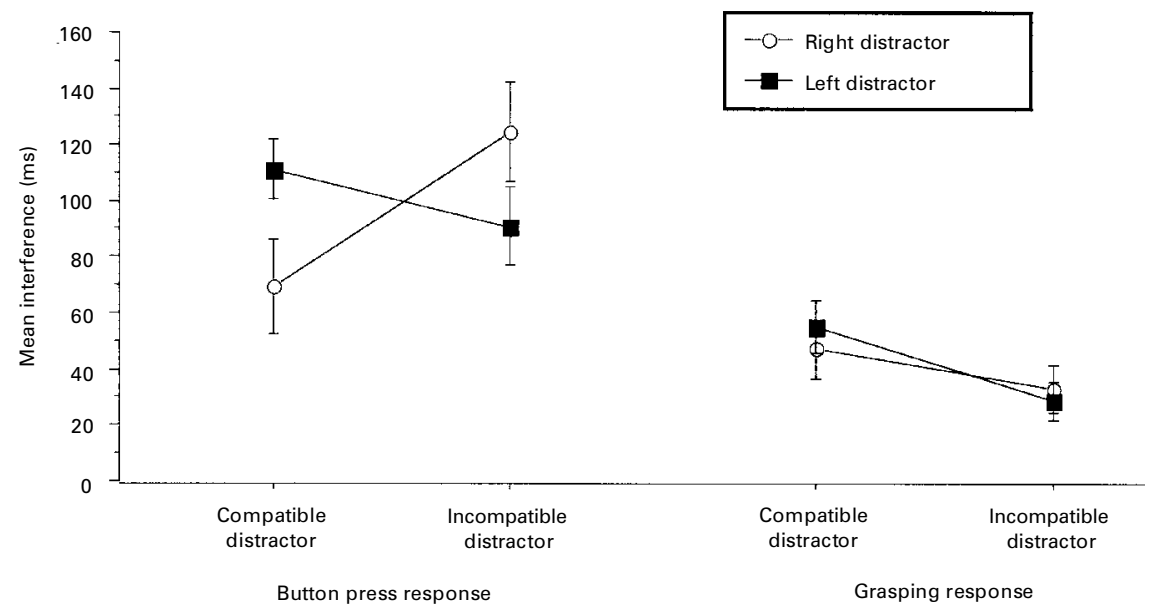

Figure 5. Interference in ms (and standard error) as a function of response type (button press and grasping), distractor compatibility (compatible and incompatible), and distractor position (left and right) in Experiment 1.

distractors caused more interference than compatible distractors. The most interesting results are the two significant three-way interactions of Response type $\times$ Target compatibility $\times$ Distractor compatibility, and Response type $\times$ Distractor compatibility $\times$ Distractor position. In the grasping task, as predicted, compatible distractors were always more interfering than incompatible distractors, regardless of target compatibility. In the button-press task, in contrast, incompatible distractors were more interfering than compatible distractors when the target was compatible, whereas a suggestion of the opposite tendency (compatible distractors slightly more interfering than incompatible distractors) was observed when the target was incompatible. The pattern of interference effects found in the button-press task can be interpreted as consistent with a response congruency effect: Greater interference when target and distractors are associated with different button-press responses than when they are associated with the same button-press response. Thus, it is easier to programme an abstract button-press response when both stimuli in the array call for the same response than when they call for conflicting responses (Eriksen \& Eriksen, 1974).

The second three-way interaction indicated that in the button-press task, compatible distractors (handle on the right) yielded more interference when they were presented in the left position than in the right position. Incompatible distractors (handle on the left) tended to yield more interference when they were presented in the right position than in the left position. This effect can be explained as a perceptual salience effect. In the compatible condition, 

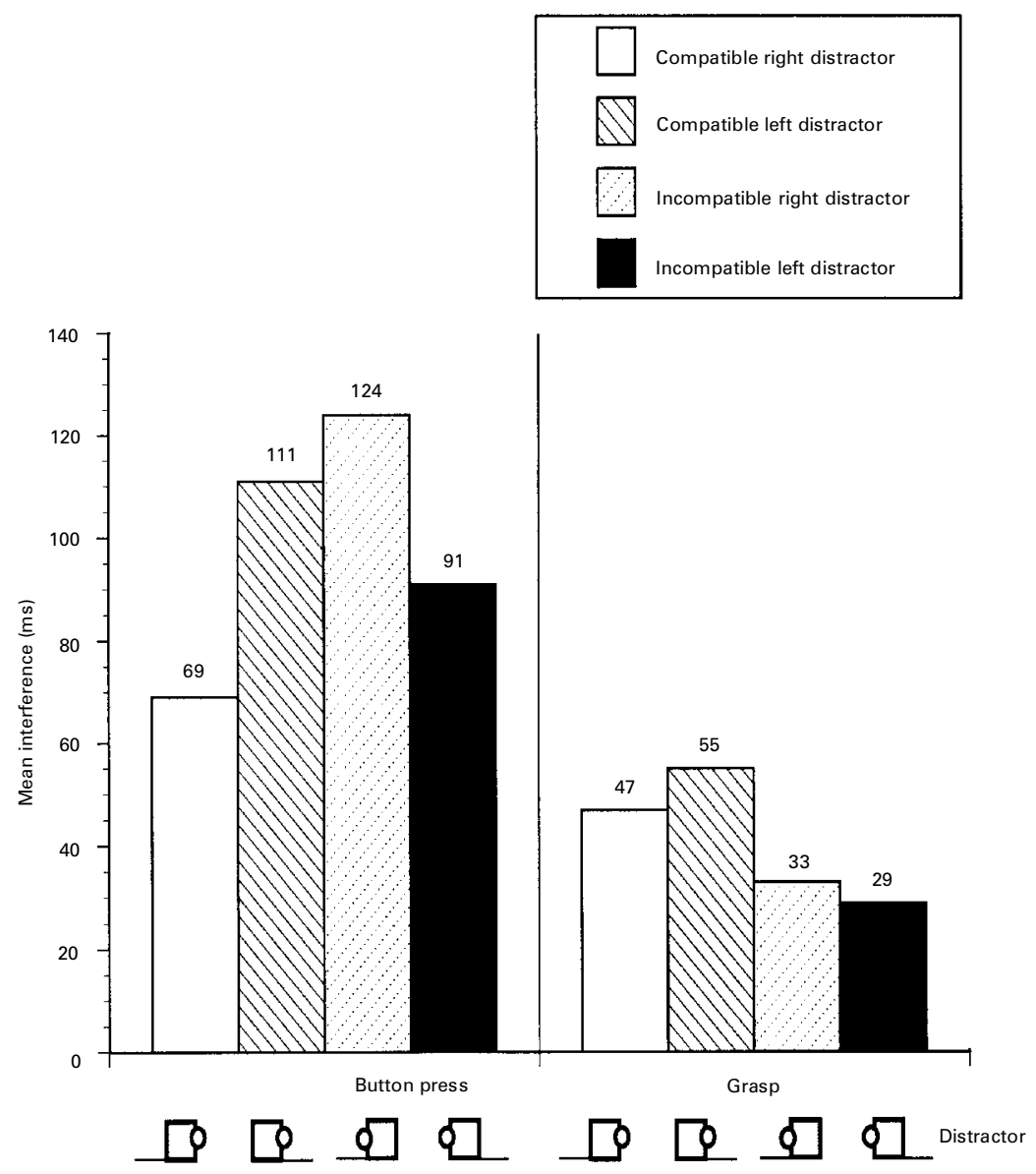

Figure 6. Interference in Experiment 1 as a function of response type, distractor compatibility, and distractor position, shown with graphic representations of stimulus configurations in each condition.

distractor handles are facing right. In this condition, left distractors will have right-facing handles close to the centre of the display, that is, close to fixation, whereas right distractors will have handles on the extreme right, far from fixation. In the incompatible condition, handles are facing left. In this case, it is the right distractors that are more interfering. This is the condition in which the handle is again in the centre. For incompatible left distractors, handles are on the extreme left of the display, and are less interfering.

To summarize, in the button-press task, response congruency and perceptual salience effects guide the pattern of interference, whereas in the grasping task only affordance variables in the distractors (compatibility between handle 
position and acting hand) modulate interference effects. Thus, selection for action is influenced by the nature of the action, i.e., attention to objects in the array is allocated as a function of the actions intended. When the action plan calls for actual interaction with objects, the features of all objects in the array affording the intended action are highlighted by attention.

Surprisingly, in this experiment we did not find an effect of distractor position in the grasping task. We expected right distractors to be more interfering than left distractors, especially (or solely) in the grasping task. However, the only effect of position was in the button-press task. Here, distractor position interacted with distractor compatibility, indicating differential perceptual salience of distractor handles as a function of their position in the display. In the grasping task, none of the effects involving position were significant $(p>.15)$.

A possible effect of distractor position is suggested by the Target compatibility $\times$ Distractor position interaction, which only approached significance, $p=.06$. This effect indicated a trend toward greater interference from right distractors when the target handle faced right and greater interference from left distractors when the target handle faced left. This result suggests that distractors on the same side as the target handle yielded more interference than distractors that are on the opposite side. This effect is likely to arise after target selection, because it depends on the position of the target handle, but not on the position of distractor handle, $p>.9$, and is independent of response type, $p>$.9. Thus, it is possible that after target selection and before response execution, objects that are close to the relevant response attribute (in this case, the target handle) become particularly salient and yield more interference than objects that are far from the response attribute.

Methodological differences may explain the discrepancy between the results of this experiment and those reported by Tipper et al. (1992). First, Tipper and colleagues used a pointing response in their study, whereas in the present experiment we used a grasping response. There is evidence that grasping and pointing responses differ in their degree of pre-programming, with grasping relying more on on-line control than pointing (Carnahan, Goodale, \& Marteniuk, 1993), and it is possible that such differences may affect the pattern of interference observed. Second, the stimuli used in the two studies were quite different: Tipper and colleagues used simple buttons arranged in a two-dimensional array, whereas we used more complex, threedimensional stimuli. We will pursue the implications of this point later. Third, in this experiment participants were asked to respond to either the left or the right side of the target object, depending on the position of the handle, whereas in Tipper et al.'s study the reaching always terminated at the centre of the target stimulus. It is possible that one or more of these factors are responsible for the absence of a distractor position effect in this experiment, but further investigation is required to address these issues. 


\section{EXPERIMENT 2 Effects of presence of handles}

In Experiment 1, we showed that distractors with good affordances for grasping yielded more interference than distractors with bad affordances, but that this was true only when the task required reaching to and grasping the handle of the target, and not when it required pressing a button on a keypad. There were two main differences between the button-press and the grasping response in Experiment 1. First, the grasping response required reaching for the target object, whereas the button-press response required pushing a button close to the hand, in the absence of any reaching movement. Second, grasping required acting on the target handle, whereas button pressing required participants to map the position of the target handle onto the two response keys following an arbitrary rule. Therefore, differences in distractor interference could have been caused by (1) the presence/absence of the reaching movement, (2) the presence/absence of manipulation of the handle attribute of the target object, or (3) both.

In Experiment 2, participants were asked to use three different motor responses: Button press, reaching and grasping, and reaching and pointing. If affordance-based interference is associated with the presence of a reaching movement but not with the type of distal manipulation, we should find differences in distractor interference between button press and the two reaching responses (grasping and pointing), but not between the two reaching responses. If affordance-based interference is associated with manipulation of the handle rather than with the reaching component, we should find a difference between button press and pointing, on the one hand, versus grasping, on the other. If both proximal and distal component of the movement influence distractor interference, we should find that all three responses are associated with different interference patterns.

\section{Methods}

In Experiment 2 we used the same stimuli and target selection criterion (blue colour) as in Experiment 1. The target object was a blue cup and the distractor a purple cup. In the handle present condition, the cups were presented with the handle facing right. In the handle absent condition, the cups were presented with the handle in the back, invisible to the subject. The three distractor conditions were handle present, handle absent, and no-distractor. The no-distractor condition was used as a baseline to compute interference scores. Two different responses were used: Reaching (pointing and grasping) and button press. In the reaching tasks, participants were asked to grasp the handle if the blue target cup had a handle or to point to the body of the cup if the blue cup did not have a handle. In the button-press task, participants were asked to press a button if the 
blue target cup had a handle and a different button if the blue cup did not have a handle. The button-press task was thus analogous to the grasp task with respect to the selection of a response based on the presence or absence of a critical feature in the target.

Half the participants began the experiment by responding to handled targets with the upper button and half started by responding to handled targets with the lower button; this mapping was switched half-way through the experiment.

The design included four factors: Response type (button press and reaching), target type (cup with handle and cup without handle), distractor type (cup with handle, cup without handle, and no distractor), and distractor position (left and right). Therefore, the design included 24 cells, 12 for each response type.

The experimental session was divided in four 96-trial blocks - two reaching blocks and two button-press blocks. The two blocks for each task were run consecutively and the order of the two tasks (reaching first vs button press first) was counterbalanced across subjects. A short block of practice was presented before each task.

\section{Results}

Table 2 shows the mean response latencies in distractor and no-distractor conditions in this experiment. Two comparisons are relevant. The first is between the button-press vs reaching tasks, and the second is the comparison between pointing and grasping responses within the reaching task. Therefore, we carried out two different ANOVAs. The first analysis compared reaching versus button press, and the second analysis included only the reaching task and examined differences in grasping versus pointing. Significance values of post hoc comparisons were calculated using two-tailed paired $t$-tests.

Button press versus reaching. We carried out a four-way repeated measures ANOVA with interference scores that included the following factors: (1) Response type (button press vs reaching), (2) target type (handle vs nohandle cup), (3) distractor type (handle vs no-handle cup), and (4) distractor position (left and right).

The main effect of response type was significant, $F(1,13)=9.73, \mathrm{MSe}=$ $3404.83, p<.01$, indicating greater interference in the button-press than in the reaching task (72 and $47 \mathrm{~ms}$, respectively). Distractor type was also significant, $F(1,13)=12.52, M S e=1779.77, p<.005$. Handle distractors were more interfering than no-handle distractors (69 and $49 \mathrm{~ms}$, respectively). The Target type $\times$ Distractor type interaction was highly significant, $F(1,13)=23.16, M S e=$ $1663.80, p<.0005$. This interaction indicates that handle distractors were more interfering when the target had no handle than when the target had a handle 
TABLE 2a

Mean response latencies in distractor and no-distractor conditions of Experiment 2

Button press

\begin{tabular}{|c|c|c|c|c|c|c|}
\hline \multirow[b]{2}{*}{ Target type } & \multicolumn{3}{|c|}{ Right } & \multicolumn{3}{|c|}{ Left } \\
\hline & Handle & No handle & No distractor & Handle & No handle & No distractor \\
\hline $\begin{array}{l}\text { Handle } \\
\text { target }\end{array}$ & 829 & 856 & 767 & 947 & 912 & 865 \\
\hline $\begin{array}{l}\text { No handle } \\
\text { target }\end{array}$ & 940 & 894 & 830 & 957 & 899 & 868 \\
\hline
\end{tabular}

TABLE 2b

\begin{tabular}{|c|c|c|c|c|c|c|}
\hline \multirow[b]{3}{*}{ Target type } & \multicolumn{6}{|c|}{ Grasp } \\
\hline & \multicolumn{3}{|c|}{ Right } & \multicolumn{3}{|c|}{ Left } \\
\hline & Handle & No handle & No distractor & Handle & No handle & No distractor \\
\hline $\begin{array}{l}\text { Handle } \\
\text { target }\end{array}$ & 925 & 943 & 879 & 893 & 907 & 862 \\
\hline $\begin{array}{l}\text { No handle } \\
\text { target }\end{array}$ & 969 & 927 & 885 & 893 & 853 & 839 \\
\hline
\end{tabular}

(84 and $55 \mathrm{~ms}$, respectively), $t(13)=-2.11, p=.05$, whereas no-handle distractors were more interfering when the target had a handle than when the target had no handle (61 and $38 \mathrm{~ms}$, respectively), $t(13)=2.29, p<.05$.

Both the two-way interaction Distractor position $\times$ Distractor type, $F(1,13)=8.69, \quad M S e=583.84, p<.02$, and the three-way interaction Response type $\times$ Distractor position $\times$ Distractor type, $F(1,13)=4.90$, $M S e=897.33, p<.05$, were significant (Figures 7 and 8).

In the button-press task, handle distractors were much more interfering than no-handle distractors when they were on the left ( 85 and $39 \mathrm{~ms}$, respectively), $t(13)=4.19, p=.001$, but they yielded comparable interference when they were on the right (86 and $77 \mathrm{~ms}$, respectively), $t(13)=1.047, p>.3$. In the reaching task, handled distractors were always more interfering than no-handle distractors, $t(13)=2.22, p<.05$, and there was a tendency for right distractors to interfere more than left distractors, $t(13)=1.85, p=.09$. These two variables did not interact $(F<1)$.

Pointing vs grasping. To examine whether handled distractors were more interfering when participants had to grasp than when they had to point, a separate analysis of the reaching task was carried out. An affordance-related 


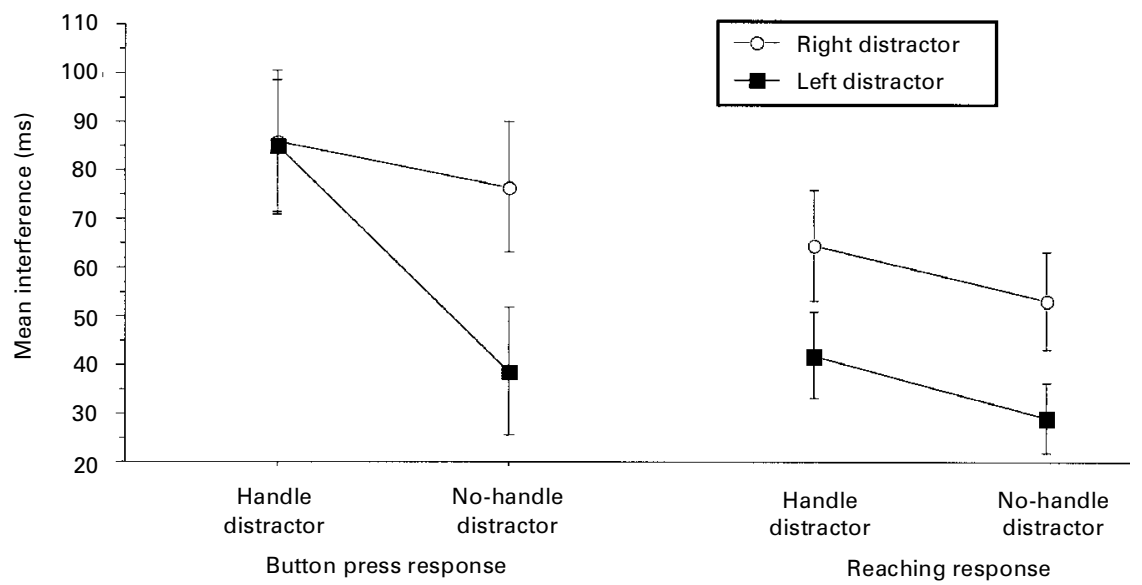

Figure 7. Interference in ms (and standard error) as a function of response type (button press and reaching), distractor type (handle and no-handle), and distractor position (left and right) in Experiment 2 .

interference effect would manifest as an interaction between target type and distractor type. In particular, we expected that when the target had a handle, and thus the response required grasping the handle, we would find more interference from handle distractors than when the target did not have a handle and the task required pointing to the cup.

The Target type $\times$ Distractor type interaction was indeed significant in this analysis, $F(1,13)=21.21, M S e=1086.96, p=.0005$, but the pattern of interference was different than predicted (Figure 9). The interaction indicates a strong response congruency effect, similar to what was observed in the previous analysis, in which handle distractors were more interfering than nohandle distractors when the targets had no handles, and thus the required response was pointing (69 and $28 \mathrm{~ms}$, respectively), $t(13)=4.15, p=.0025$, and no-handle distractors were more interfering than handle distractors when the target had handles, and the required response was grasping (54 and $38 \mathrm{~ms}$, respectively), $t(13)=-2.62, p<.025$. This is opposite to what we had predicted.

\section{Discussion}

In Experiment 2, we again found differences in the pattern of distractor interference as a function of response type. In particular, when the task required participants to reach for the target, handle distractors were more interfering than nohandle distractors and there was a tendency for right distractors to be more interfering; there was no interaction between these two variables. When the 


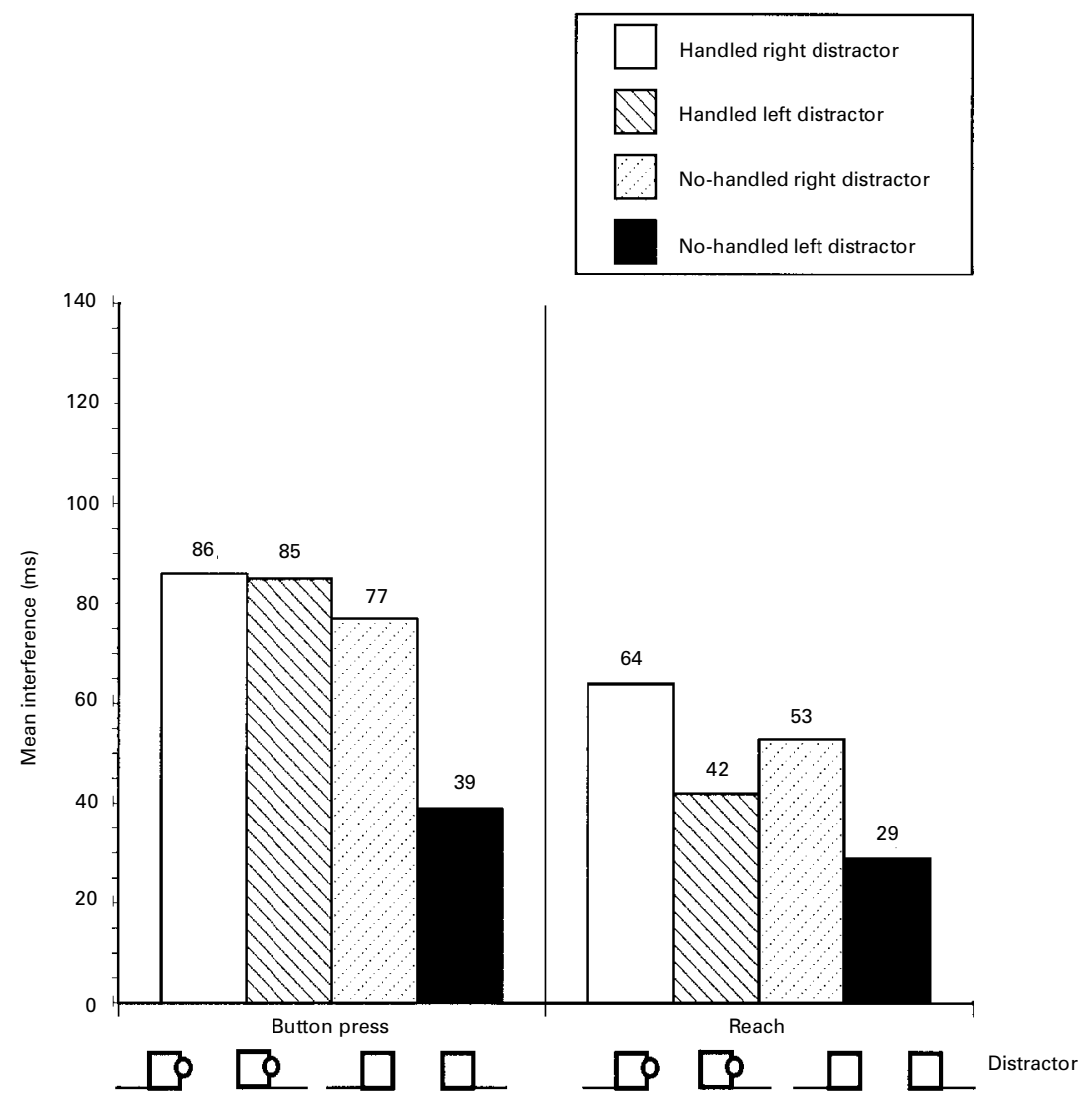

Figure 8. Interference in Experiment 2 as a function of response type, distractor type, and distractor position, shown with graphic representations of stimulus configurations in each condition.

task required participants to press a button, right distractors yielded a similar amount of interference whether or not they had handles (86 and $77 \mathrm{~ms}$, respectively), whereas left distractors were much more interfering when they had handles than when they did not have handles ( 85 and $39 \mathrm{~ms}$, respectively). This is likely a perceptual effect similar to what we observed in Experiment 1 . When the distractor handle is central and close to fixation (cup in left position with handle facing right) it yields greater interference.

This pattern of results confirms that different variables influence distractor interference depending on whether the task requires reaching for the target or pressing a button associated arbitrarily with the target attribute. In the reaching task, affordance and action-centred effects predominated: Handle distractors were always more interfering than no-handle distractors, and right distractors 


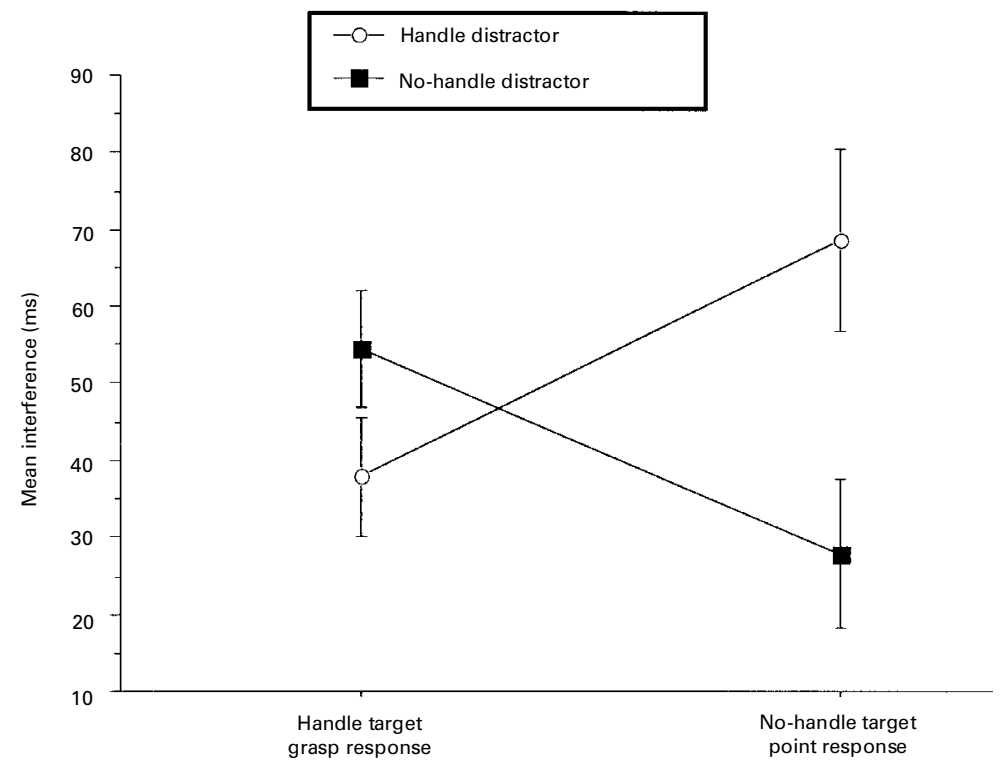

Figure 9. Interference in ms (and standard error) as a function of target type (handle and no-handle) and distractor type (handle and no-handle) in the reaching condition of Experiment 2.

tended to cause greater interference than left distractors. In the button-press task, perceptual elements (such as salience of a handle presented at fixation) were more influential in modulating interference.

We expected to find a differential effect of handle distractors depending on the type of reaching response (grasping vs pointing), but this was not the case. The results of Experiment 2 suggest that affordances influence distractor interference any time participants must reach for the target object, regardless of the distal component of the action. However, before accepting this conclusion, we must consider two factors that may have reduced the possibility of observing differential effects of pointing and grasping in this experiment.

First, the experiment required selection of an appropriate action (pointing or grasping) subsequent to target identification and contingent upon the features of the target. This means that although participants knew in advance that they had to reach for the target object, they did not know whether they had to grasp or point to the target until the target object was selected. If it is the case that an action plan must first be selected to influence target selection processes, it would then not be surprising that, under the present conditions, we would observe a difference in patterns of interference as a function of the presence or absence of the reaching component, but not with changes in the manipulation component. 
Second, cups are not likely to afford pointing and grasping equivalently. Cups afford grasping, regardless of whether handles are present or absent, in part because of their physical features (e.g., hand-sized curvature), and in part because of the over-learned response of grasping cups. On the other hand, neither learned use nor object shape suggest an association between cups and the action of pointing. Therefore, although grasping may receive automatic activation from the cup's features as well as from task instructions ("grasp the target if it has a handle"), pointing receives activation only from the task instruction ("point to the target if it does not have a handle").

This observation suggests that when the target has a handle, selecting the grasping response should be relatively easy, and a no handle distractor should yield minimal interference. Thus, with handle targets we should find interference to be similar whether the distractor is associated with the same response (handle distractor) or opposite response (no-handle distractor). On the contrary, when the target has no handles, selecting the pointing response should be relatively difficult in the presence of a handle distractor that activates a grasping response. The results of the Target type $\times$ Distractor type interaction, described previously in terms of a response congruency effect, follow this pattern. In the handle target/grasp response condition, no handle distractors caused $16 \mathrm{~ms}$ more interference than handle distractors. In the no-handle target/point condition, in comparison, the relative interference caused by handle distractors as compared to no-handle distractors was much greater $(41 \mathrm{~ms})$. This difference between the two conditions is significant, $t(13)=-2.22, p<.05$ (Figure 9). In other words, the response congruency effect is largely "driven" by the no handle target/handle distractor condition.

To maximize the chances of finding differences in distractor interference in pointing versus grasping responses, we reasoned that the experimental paradigm must be changed in two ways. First, the type of motor response should be blocked, so that the appropriate distal action is selected before target selection and can influence distractor processing. Second, we should use two types of objects: Objects that have good affordances for grasping, and objects that have good affordances for pointing. The aim of this manipulation would be to allow equivalent activation of grasping and pointing actions by different objects in the array.

\section{EXPERIMENT 3}

\section{Button interference versus handle interference}

Experiment 3 had the same purpose as Experiment 2, but we made some modifications to maximize the possibility of finding a differential effect of distractor affordances as a function of reaching response (pointing vs grasping). The first modification was to block the motor response required so that target selection occurred after action selection. The second was to use objects with clear 
affordances: Button-switches affording pointing but not grasping, and drawer and door handles affording grasping but not pointing (Figure 10).

To ensure that affordances and not visual similarity were influencing interference, in each affordance set we used two perceptually different objects. The two objects that afforded pointing were a round button (doorbell) and a long button (light-switch), and the two objects that afforded grasping were a round handle and a long handle. In each trial, the target afforded either grasping or pointing, the distractor afforded either grasping or pointing, and the two objects were always perceptually dissimilar. For example, if the target was a long handle, the distractor could be a round button or a round handle.

Our predictions were that in the grasping blocks, distractors that afforded grasping would be more interfering than distractors that afforded pointing. In the pointing blocks, distractors affording pointing should interfere more than distractors affording grasping. As in the prior experiments, a button-press task served as a non-reaching control.

\section{Methods}

In this experiment, the target could be one of four objects created by the crossing of two factors: Shape (round and long) and affordance (buttons affording pressing and handles affording grasping; see Figure 7). Each object

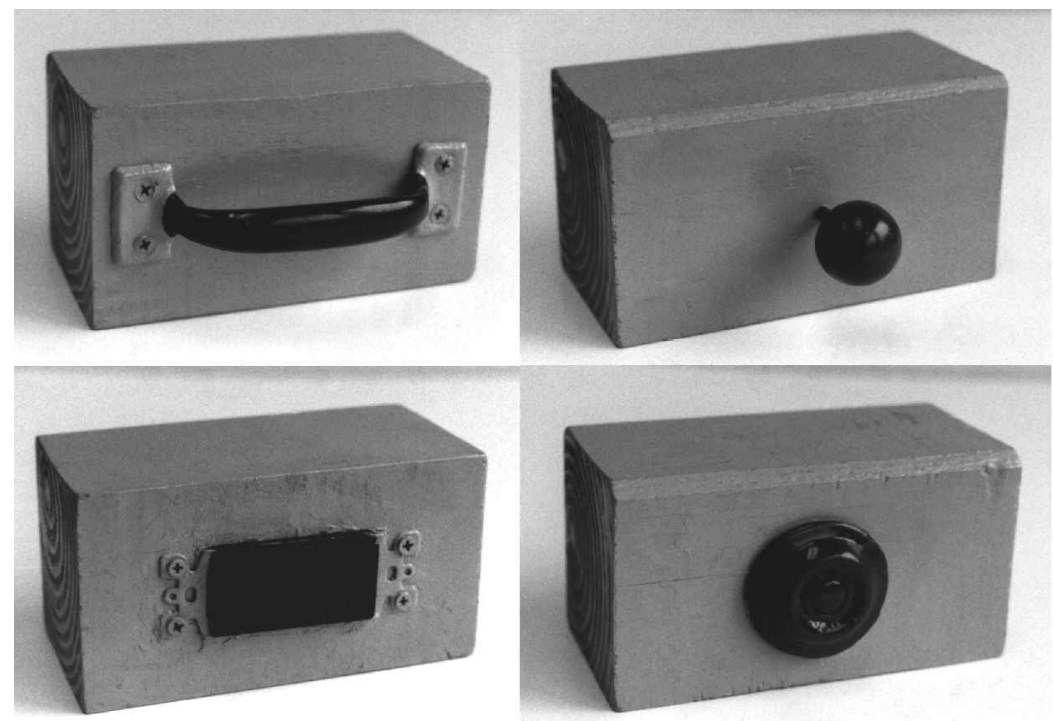

Figure 10. Stimuli used in Experiment 3. On the left are shown the two long stimuli and on the right the two round stimuli. The upper row shows the two handles and the lower row the two buttons. 
was mounted on a wooden block of $3 \frac{1}{2}$ by $3 \frac{1}{2}$ by $6 \frac{1}{2}$ inches. The long handle was $4 \frac{3}{8}$ inches long, $3 / 4$ inches wide, and protruded from the block $7 / 8$ inches. The round handle had a diameter of $1 \frac{1 / 2}{2}$ inches and protruded from the block $3 / 4$ inch. The long button was $2 \%$ inches long, $1 \frac{1}{4} 4$ inches wide, and protruded from the block $1 / 4$ inch. The round button had a diameter of $5 / 8$ inches and sat in a base of $2 \frac{1}{4}$ inches in diameter; the button-and-base ensemble protruded from the block $1 / 2$ inch.

Three distractor conditions were used in this experiment: Button distractor, handle distractor, and no distractor. The no-distractor condition served as a baseline to compute interference. The experiment was run in two experimental sessions over two different days. In each session, three blocks of trials were run: A button-press block, a pointing block, and a grasping block. Each block comprised 96 trials. The order of the blocks was counterbalanced across subjects. As in prior experiments, the target object was selected on the basis of colour (blue). In the reaching conditions, participants were instructed to grasp or point to the blue target object, depending upon the block. Thus, for example, in the grasp blocks, subjects were required to grasp blue handles and blue buttons, and the buttons had a relatively poor affordance for grasping. In the button-press condition, the task required participants to press the key corresponding to the position (left or right) of the blue target object. As in previous experiments, the button-press condition was analogous in some respects to the reach conditions. In this experiment, both button-press and reaching conditions had blocked action parameters, and response selection concerned indication of the location of the target object.

\section{Results}

Table 3 shows the mean response latencies in distractor and no-distractor conditions in this experiment. Two repeated measures ANOVAs were carried out on the interference data. The first analysis examined interference data from the three response types (button press, grasping, and pointing). The goal of this analysis was to examine whether there was any difference in interference as a function of the type of response. Because the aim of this experiment was to investigate whether different patterns of interference would be observed for reaching responses with different distal components (point vs grasp), the second ANOVA analysed interference data in pointing and grasping conditions. Significance values of post hoc comparisons were calculated using paired $t$-tests.

All response types. Interference scores were analysed in a four-way ANOVA comprising response type (button press, grasping, and pointing), target type (button and handle), distractor type (button and handle), and distractor position (left and right). Only one effect reached significance. The 
TABLE 3a

Mean response latencies in distractor and no-distractor conditions of Experiment 3

Button press

\begin{tabular}{|c|c|c|c|c|c|c|}
\hline \multirow[b]{2}{*}{ Target type } & \multicolumn{3}{|c|}{ Right } & \multicolumn{3}{|c|}{ Left } \\
\hline & Button & Handle & No distractor & Button & Handle & No distractor \\
\hline Button target & 970 & 1002 & 919 & 974 & 924 & 871 \\
\hline Handle target & 980 & 978 & 935 & 1001 & 939 & 865 \\
\hline
\end{tabular}

TABLE 3b

\begin{tabular}{|c|c|c|c|c|c|c|}
\hline \multirow[b]{3}{*}{ Target type } & \multicolumn{6}{|c|}{ Grasp } \\
\hline & \multicolumn{3}{|c|}{ Right } & \multicolumn{3}{|c|}{ Left } \\
\hline & Button & Handle & No distractor & Button & Handle & No distractor \\
\hline Button target & 1147 & 1188 & 1105 & 1082 & 1096 & 1014 \\
\hline Handle target & 1098 & 1106 & 1042 & 1019 & 1028 & 931 \\
\hline
\end{tabular}

TABLE 3c

\begin{tabular}{|c|c|c|c|c|c|c|}
\hline \multirow[b]{3}{*}{ Target type } & \multicolumn{6}{|c|}{ Point } \\
\hline & \multicolumn{3}{|c|}{ Right } & \multicolumn{3}{|c|}{ Left } \\
\hline & Button & Handle & No distractor & Button & Handle & No distractor \\
\hline Button target & 1148 & 1164 & 1079 & 1063 & 1077 & 991 \\
\hline Handle target & 1103 & 1122 & 1043 & 1034 & 1031 & 953 \\
\hline
\end{tabular}

Response type $\times$ Distractor type interaction was highly significant, $F(1,17)=17.09, M S e=8887.33, p<.0001$ (Figure 11). In the button-press task, button distractors were more interfering than handle distractors (83 and $63 \mathrm{~ms}$ for button interference and handle interference, respectively), $t(18)=2.84, p<.02$ ), whereas the opposite pattern was observed in the two reaching tasks: Handle distractors were more interfering than button distractors for the grasping response ( 81 and $64 \mathrm{~ms}$, respectively), $t(18)=-2.22, p<.05$, and showed a strong tendency in the same direction for the pointing response (82 and $70 \mathrm{~ms}$, respectively), $t(18)=-1.90 . p=.07$.

Pointing vs grasping. We carried out a second four-way ANOVA in which task (pointing and grasping), target type (handle and button), distractor 


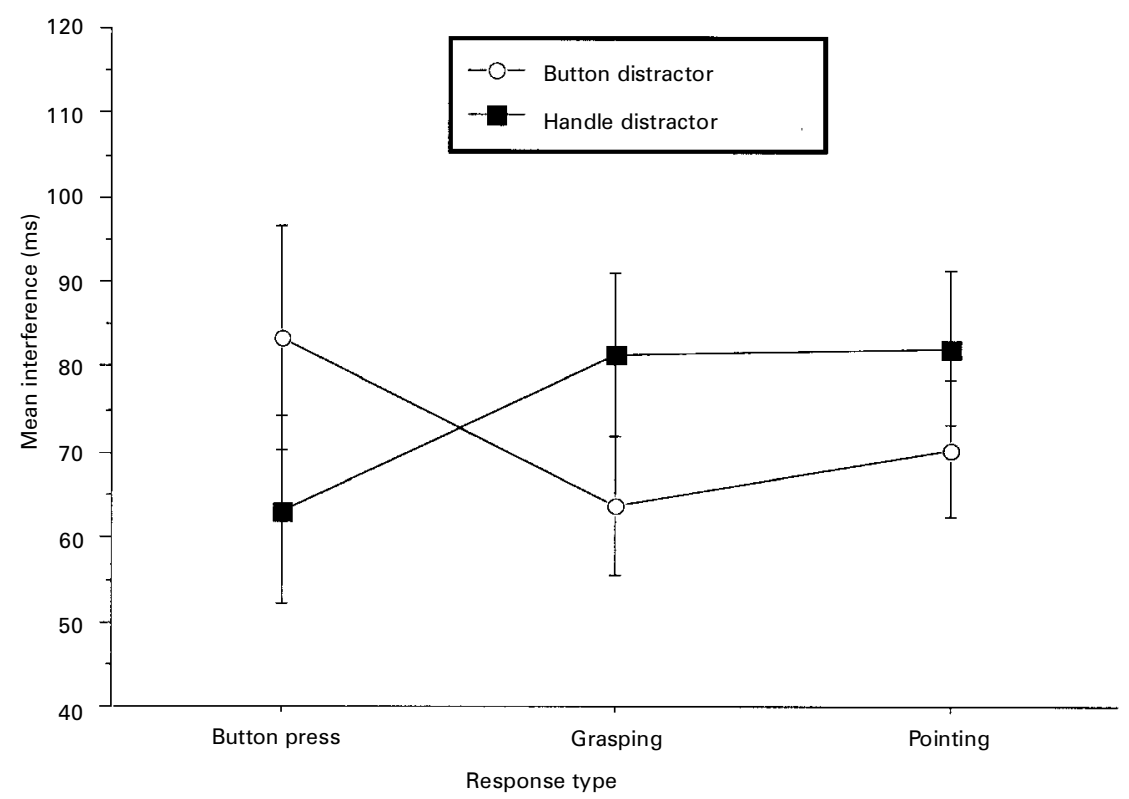

Figure 11. Interference (in $\mathrm{ms}$ ) as a function of response type (button press, grasping, and pointing) and distractor type (button and handle).

type (handle and button), and distractor position (left and right) were factors. The only significant effect in this analysis was distractor type, $F(1,17)=5.23$, $M S e=3027.18, p<.04$, indicating that handle distractors were more interfering than button distractors ( 82 and $67 \mathrm{~ms}$, respectively) in both the pointing and grasping tasks. Distractor type did not interact with response type $(F<1$, $p<.3)$.

\section{Discussion}

In Experiment 3 we examined interference effects in three tasks: Button press, reaching and pointing, and reaching and grasping. Button press and pointing have a similar distal component, but button press lacks the reaching component that the pointing task requires. Pointing and grasping both have a reaching component, but differ in the final hand shape used to interact with the object.

The interpretation of Experiment 3 is relatively straightforward. The highly significant interaction between response type and distractor type indicates that button distractors yielded greater interference than handle distractors in the button-press task, whereas handle distractors yielded more interference in the two tasks that required reaching for the target (grasping and pointing). A direct 
comparison between pointing and grasping confirmed that in both tasks, handle distractors were more interfering than button distractors, but did not reveal any differences between the two response types.

Three points are worth discussing. First, the results of Experiment 3 closely replicated those of Experiment 2, indicating that reaching for an object produces a different pattern of distractor interference than pressing a button close to the hand, but that the type of distal manipulation of the target (pointing or grasping) does not influence distractor interference. This replication is particularly striking because in Experiment 3 we used different stimuli and modified the experimental procedure to maximize the chances of finding differential affordance-related interference effects associated with pointing and grasping responses. It is possible to account for these results by assuming that reaching actions, regardless of the distal component, always increase the salience of objects that afford grasping. We will pursue this further in the General Discussion.

Second, in Experiment 3 we found relatively greater interference from button as compared to handle distractors in the button-press task. Because the distal movements used in button presses and in pointing to door bells or switches are similar, it is possible that this constitutes an affordance-related effect. It is interesting to note that this is the only experiment in which we used objects that afford pointing, and the only experiments in which we found putative affordance effects for button-press responses. Thus, even with responses not requiring participants to reach and act on the target object, it may be possible to observe affordance-related effects. As discussed earlier, findings potentially relevant to this notion have been reported previously (Tucker \& Ellis, 1998).

Third, it is of note that in this experiment we did not observe the "actioncentred" interference effects reported by Tipper and colleagues (1992). The effect of distractor position approached significance in the overall analysis, $F(1,17)=3.94, p=.063$, but the trend was in the opposite direction: Distractors close to the acting hand tended to be less interfering than distractors far from the acting hand (63 and $85 \mathrm{~ms}$, respectively). Furthermore, there was no hint of an interaction between distractor position and response type $(F<1$, $p>$.4).

As already mentioned, it is possible that differences in the motor responses and stimuli used in these studies are responsible for this discrepancy. It is particularly interesting that we did not observe the distractor position effect in the pointing blocks of our reaching task, in which the motor response was similar to that used by Tipper and colleagues. One possible explanation is based on the notion that it is the ease of response to a stimulus, relative to other stimuli in the array, that determines its salience as a distractor. The relative potency of distractors close to the hand (the "action-centred" effect) may be observed only when targets and distractors are two-dimensional shapes of no naturalistic 
relevance to the actor (e.g., coloured lights). Under such circumstances, potential actions to all objects are similar, and location is a relatively salient feature of the stimuli. In contrast, when familiar objects are used, other object features such as affordances or pragmatic characteristics of the objects (Jeannerod, 1994) may determine the ease of response to the objects, and thus have relatively stronger effects on the pattern of distractor interference. In this case, the degree to which object affordances match planned actions, rather than relative location, may become dominant in determining interference effects, and spatial effects concerning the relationship of the responding hand and objects in the array will be difficult to observe. This is a speculative proposal, but it may be useful in informing future research.

\section{GENERAL DISCUSSION}

In this study, our goal was to examine the influence of affordances and action parameters on selection for action. To maximize the possibility that we would observe affordance effects, we used familiar objects (cups with and without handles in Experiments 1 and 2; drawer handles, doorbells, and light-switches in Experiment 3) associated naturalistically with grasping and pointing responses. To examine the effect of action parameters, we varied the type of motor response required. In Experiment 1, we assessed the hypothesis that in a task requiring reaching to and grasping a target cup, the interference caused by distractors with handles would be modulated by the position of the handle relative to the responding hand. In Experiment 2, we tested the hypothesis that the type of reaching response to target cups (pointing vs grasping) may influence the interference caused by distractor cups with and without handles. In Experiment 3, we assessed whether affordance-related interference effects are enhanced when objects strongly afford their associated actions and when these actions can be pre-programmed.

In all three experiments we found task effects: Button press and reaching actions produced different pattern of interference. In Experiments 1 and 2, we used objects that afford grasping (cups), and found that button-press actions were associated with perceptual effects (greater interference when critical features were presented close to fixation) and response congruence effects (greater interference by distractors associated with different responses than targets). In contrast, reaching and grasping was associated with affordanceinterference effects (greater interference by compatible distractors in Experiment 1; greater interference by handled distractors in Experiment 2). In Experiment 3, we used objects that afford grasping (handles) and objects that afford pointing (door bells and light-switches), and found that reaching actions were associated with affordance-interference effects (greater interference from handled distractors). In contrast, button-press actions were more vulnerable to interference from distractors affording pointing than from distractors affording 
grasping. Thus, selection for action is influenced by the nature of the action, that is, attention to objects in the array is allocated as a function of the actions intended. When the action plan calls for actual interaction with objects, the features of all objects in the array affording action are highlighted by attention. The handles of target and distractor cups are attended differently depending upon whether one intends to reach to or merely locate the target.

Contrary to our predictions, we failed to find a difference in interference as a function of the precise hand posture required (grasping vs pointing). Instead, in all tasks requiring reaching to objects in the array, distractors affording grasping (handle distractors) generally caused more interference than distractors not affording grasping, regardless of the hand posture afforded by the target (Experiments 2 and 3) or required by the blocked nature of response instructions (Experiment 3). One possibility is that the asymmetric affordanceinterference effects observed (strong interference by distractors affording grasping and weak interference by distractors affording pointing in both grasping and pointing tasks) may reflect differences in the processing or representation of objects affording grasping as compared to pointing actions. Given that relatively large portions of the dorsal visual processing stream in monkey and man (e.g., areas AIP and F5 and F6 in the monkey) are devoted to processing various kinds of grasp actions (e.g., precision grip, power grip), it is possible that the grasping response is dominant over other types of manual responses, and that objects affording grasping, in part because of the sheer magnitude of their neural representation, are strong competitors for the control of action irrespective of the manual response required (e.g., Grafton, Arbib, Fadiga, \& Rizzolatti, 1996; MacKay, 1992; Rizzolatti et al., 1988; Sakata \& Taira, 1994; Stein, 1992).

Additionally of interest are the data from Experiment 3, which revealed that button distractors in the array were highly interfering when the response was a button press on the table-top. We suggest that the emergence of this effect may have been possible because of differences between this experiment and the previous experiments. In this experiment, it was not possible to observe "response congruency" effects as in Experiments 1 and 2, because subjects responded on the basis of the location of the target (left or right), and there was never a case in which both the target and distractor called for the same response. Second, unlike in previous experiments, the stimuli were symmetrical (i.e., without handles on the left or right), so it was not possible to observe perceptual salience effects (e.g., handles close to initial fixation at midline). Finally, this was the only experiment in which we used stimuli that had a strong affordance for pointing. It is not clear which of these factors are instrumental in revealing the strong interference by buttons in the button-press task; this will be an interesting question for future investigations.

Several investigators (Castiello, 1998, 1999; Tucker \& Ellis, 1998) have suggested that objects in the array automatically activate responses associated 
with them, even in the absence of the subject's intention to act. This phenomenon has been termed a "visuomotor priming" effect (Craighero, Fadiga, Rizzolatti, \& Umiltà, 1999). Our data speak to a phenomenon that is in some respects opposite to this; namely, whether preparation and planning of an action affects the features of objects highlighted by attention. Our data suggest that object affordances are indeed attended (and hence, activate their associated responses) differently depending upon the intentions and plans of the actor. This notion accords well with the "premotor theory of attention" proposed by Rizzolatti and colleagues (Rizzolatti \& Camarda, 1977; Rizzolatti, Riggio, Dascola, \& Umiltà, 1987). On this theory, activity of neural circuits programming motor plans produces a shift of attention to the spatial regions where the action is to be executed. In other words, visual attention to a particular location comprises facilitation of neurons involved in preparing and directing actions to that part of space. The present data suggest an extension of the premotor account: That the preparation of an action influences the particular features of objects that are attended. Thus, in tasks requiring action upon objects in the array, it is the relationship between objects and potential actions that guide interference effects. In contrast, if no actions upon the objects are planned, as is the case in many traditional distractor interference studies, it is the relationship between target and distractor objects (e.g., relative locations, relative discriminability; Duncan \& Humphreys, 1989) that influences the pattern of interference.

\section{REFERENCES}

Allport, D.A. (1987). Selection for action: Some behavioral and neuropsychologica 1 considerations of attention and action. In H. Heuer \& A.F. Sanders (Eds.), Perspectives on perception and action (pp. 395-419). Hillsdale, NJ: Lawrence Erlbaum Associates, Inc.

Carnahan, H., Goodale, M.A., \& Marteniuk, R.G. (1993). Grasping versu s pointing and the differential use of visual feedback. Human Movement Science, 12(3), 219-234.

Castiello, U. (1996). Grasping a fruit: Selection for action. Journal of Experimental Psychology: Human Perception and Performance, 22, 582-603.

Castiello, U. (1998). Attentional coding for three-dimensional objects and two-dimensional shapes: Differential interference effects. Experimental Brain Research, 123(3), 289-297.

Castiello, U. (1999). Mechanism s of selection for the control of hand action. Trends in Cognitive Sciences, 3(7), 264-271.

Craighero, L., Fadiga, L., Rizzolatti, G., \& Umiltà, C. (1999). Action for perception: A motorvisual attentional effect. Journal of Experimental Psychology: Human Perception and Performance, 25(6), 1673-1692.

Duncan, J., \& Humphreys, G.W. (1989). Visual search and stimulus similarity. Psychological Review, 96, 433-458.

Eriksen, B.A., \& Eriksen, C.W. (1974). Effects of noise letters upon the identification of a target letter in a nonsearch task. Perception and Psychophysics, 16, 143-149.

Gibson, J.J. (1979). The ecological approach to visual perception. Boston: Houghton-Mifflin . 
Grafton, S.T., Arbib, M.A., Fadiga, L., \& Rizzolatti, G. (1996). Localization of grasp representations in humans by positron emission tomography: 2 . Observation compared with imagination. Experimental Brain Research, 112(1), 103-111.

Jeannerod, M. (1994). Object oriented action. In K.M.B. Bennet \& U. Castiello (Eds.), Insights into the reach and grasp movement (pp. 3-15). Amsterdam: Elsevier.

Kornblum, S., Hasbroucq, T., \& Osman, A. (1990). Dimensional overlap: Cognitive basis for stimulus-response compatibility - a model and taxonomy. Psychological Review, 97, $253-270$.

MacKay, W.A. (1992). Properties of reach-relate d neuronal activity in cortical area 7A. Journal of Neurophysiolog y, 67(5), 1335-1345.

Meegan, D.V., \& Tipper, S.P. (1998). Reaching into cluttered visual environments: Spatial and temporal influences of distracting objects. Quarterly Journal of Experimental Psychology, 51A, 225-249.

Meegan, D.V., \& Tipper, S.P. (1999). Visual search and target-directe d action. Journal of Experimental Psychology: Human Perception and Performance, 25(5), 1347-1362.

Michaels, C.F., \& Stins, J.F. (1997). An ecological approach to stimulus-respons e compatibility . In B. Hommel \& W. Prinz (Eds.), Theoretical issues in stimulus-response compatibility (pp. 333-360). Amsterdam: Elsevier.

Proctor, R.W., \& Reeve, T.G. (Eds.). (1990). Stimulus-response compatibility: An integrated perspective. Amsterdam: North-Holland.

Proctor, R.W., Van Zandt, T., Lu, C.H., \& Weeks, D.J. (1993). Stimulus-response compatibility for moving stimuli: Perception of affordance s or directional coding? Journal of Experimental Psychology: Human Perception and Performance, 19(1), 81-91.

Riddoch, J.M., Edwards, M.G., Humphreys, G.W., West, R., \& Heafield, T. (1998). Visual affordance s direct action: Neuropsychologica 1 evidence from manual interference. Cognitive Neuropsychology, 5, 645-683.

Rizzolatti, G., \& Camarda, R. (1977). Influence of the presentation of remote visual stimuli on visual responses of cat area 17 and lateral suprasylvian area. Experimental Brain Research, 29(1), 107-122.

Rizzolatti, G., Camarda, R., Fogassi, L., Gentilucci, M., Luppino, G., \& Matelli, M. (1988). Functional organization of inferior area 6 in the macaque monkey: II. Area F5 and the control of distal movements. Experimental Brain Research, 71(3), 491-507.

Rizzolatti, G., Riggio, L., Dascola, I., \& Umiltà, C. (1987). Reorienting attention acros s the horizontal and vertical meridians: Evidence in favor of a premotor theory of attention. Neuropsychologia, 25(1A), 31-40.

Sakata, H., \& Taira, M. (1994). Parietal control of hand action. Current Opinions in Neurobiology, 4(6), 847-56.

Shaw, R., \& Turvey, M.T. (1981). Coalitions as models of eco-systems : A realist perspective on perceptual organization. In M. Kubovy \& J.R. Pomerantz (Eds.), Perceptual organization. Hillsdale, NJ: Lawrence Erlbaum Associates, Inc.

Stein, J.F. (1992). The representation of egocentric space in the posterior parietal cortex. Behavioral and Brain Sciences, 15, 691-700.

Tipper, S.P., Howard, L.A., \& Jackson, S.R. (1997). Selective reaching to grasp: Evidence for distractor interference effects. Visual Cognition, 4, 1-38.

Tipper, S.P., Lortie, C., \& Baylis, G.C. (1992). Selective reaching: Evidence for action-centere d attention. Journal of Experimental Psychology: Human Perception and Performance, 18, 891-905.

Tucker, M., \& Ellis, R. (1998). On the relation s between seen objects and components of potential actions. Journal of Experimental Psychology: Human Perception and Performance, 24(3), 830-846. 